\title{
Characterization of grain boundaries in CdTe polycrystalline films
}

\author{
V.V. Tetyorkin ${ }^{1}$, A.V. Sukach ${ }^{1}$, V.A. Boiko ${ }^{1}$, A.I. Tkachuk ${ }^{2}$ \\ ${ }^{I}$ V. Lashkaryov Institute of Semiconductor Physics, NAS of Ukraine, \\ 41, prospect Nauky, 03680 Kyiv, Ukraine \\ ${ }^{2}$ V. Vinnichenko Kirovograd State Pedagogical University, Kirovograd, Ukraine \\ Phone: 38 (044) 525-1813, e-mail:teterkin@isp.kiev.ua
}

\begin{abstract}
CdTe polycrystalline films with the average size of grains within the range $10 \ldots 360 \mu \mathrm{m}$ were grown on sapphire substrates by using the modified close-spaced sublimation technique. Transverse (across the film) and lateral (along the film's surface) conductivity as a function of bias voltage and temperature were measured using appropriate arrangement of contacts. The transverse conductivity exhibits ohmic behavior, whereas the lateral transport of carriers is dominated by potential barriers at the grain boundaries. The carrier concentration in the grains and the potential barrier height have been estimated. The inhomogeneous distribution of deep defects through the grains was found from the photoluminescence measurements.
\end{abstract}

Keywords: CdTe polycrystalline films, potential barrier height, carrier concentration, sapphire substrates, grain boundaries.

Manuscript received 12.05.15; revised version received 19.08.15; accepted for publication 28.10.15; published online 03.12.15.

\section{Introduction}

It is generally believed that grain boundaries in polycrystalline films dominate their physical properties. The influence of grain boundaries especially increases in polycrystalline films composed of small grains. Because of atomic structure and electronic properties of grain boundaries are not fully understand, theoretical modeling is regarded as a powerful means for investigation of physical properties inherent to polycrystalline films. A number of models were earlier developed [1,2]. Among others, Petriz's model [3] seems to be the most cited. As a rule, grain boundaries are modeled by identical topological and atomic structure as well as electronic properties. Naturally, in this case the potential barrier height is unchanged through a polycrystalline film, and its value can be estimated, for instance, from the DC conductivity measurements. However, in 'real' films atomic structure as well as electronic properties of grain boundaries may vary within a separate grain, giving rise to the barrier height fluctuation [1-3]. Moreover, bulk properties of grains, such as inhomogeneous distribution of native defects and impurities in the intragrain region, variations in the grain size and form, can also influence the barrier height. In this case, theoretical approaches developed for transport phenomena in disordered semiconductors may be useful for characterization of polycrystalline films [4-6].

CdTe thin films are widely used for manufacturing different semiconductor devices, namely: solar cells, infrared, X-ray and gamma-ray detectors for medical and industrial imaging systems, etc. [7-9]. To prepare CdTe polycrystalline films suitable for device applications, several methods as well as a range of substrate materials were used. Fabrication of ohmic contacts to $p$-CdTe with stable and reproducible characteristics still remains an 
important problem. Usually, it is achieved by formation of heavily doped region at the surface by using appropriate dopant. For instance, copper-containing contacts have been developed for $p$-CdTe which include utilization of $\mathrm{Cu}_{2} \mathrm{Te}, \mathrm{Cu}-\mathrm{Au}$ alloy, $\mathrm{ZnTe}: \mathrm{Cu}$ and $\mathrm{HgTe}: \mathrm{Cu}$ [10-13]. Note that the highest reported efficiency of solar cells $(\sim 16 \%)$ is achieved in CdS/CdTe heterostructures incorporated copper-containing contacts [9]. Unfortunately, fast migration of copper in CdTe polycrystalline films results in the long-term degradation of solar cell performance. Thus copper-free contacts have attracted great attention in CdTe technology. Several solutions were proposed earlier including usage of different materials [14-16]. To clarify the role of grain boundaries in CdTe polycrystalline films, a series of samples with different sizes of grains were prepared. Also, two arrangements of contacts were applied when measuring the DC conductivity in order to estimate the potential barrier height at the grain boundaries as well as the concentration of mobile carriers in the grains.

\section{Preparation of CdTe polycrystalline films}

Investigated in this study CdTe polycrystalline films were grown on sapphire substrates by a close-spaced sublimation technique modified in our laboratory. An undoped CdTe grown using the Bridgman technique was used as an initial source material. The two-stage deposition process has been developed for this purpose. The polycrystalline films were preliminary deposited on a glassceramic substrate. After that, they were mounted at a source container for further deposition onto sapphire substrates. Immediately before the deposition process, the sapphire substrates were etched in aqueous solution of hydrofluoric acid and washed in distilled water. The substrate holder and source container were made from a high-density graphite block. The distance between the sapphire substrate and CdTe/glassceramic source was adjusted from one to three millimeters. The developed technological module was placed in a silica ampoule and mounted in a vacuum chamber $\left(P=10^{-4} \mathrm{~Pa}\right)$ using vertical arrangement. The substrate was heated to approximately $600{ }^{\circ} \mathrm{C}$ and kept at this temperature for half an hour. After that, the substrate temperature was gradually lowered down to the required value $350 \ldots 400{ }^{\circ} \mathrm{C}$. Simultaneously, the source temperature was increased up to $500 \ldots 600^{\circ} \mathrm{C}$. By changing the growth time, the films with the average grain size ranging from 10 up to $360 \mu \mathrm{m}$ were grown. The grain size was determined using the linear intercept method [17]. The two-stage deposition process enabled us to grow polycrystalline films with columnar structure of grains.

The lateral (along the film surface) and transverse (across the film) conductivity were investigated using appropriate arrangement of contacts. As a contact material, Tl-doped $p$-PbTe with the impurity concentration of 0.8 at. $\%$ was used. The hole concentration in $p^{+}$-PbTe was of the order of $10^{19} \mathrm{~cm}^{-3}$ at room temperature. The ohmic nature of $p^{+}-\mathrm{PbTe} / p-\mathrm{CdTe}$ contacts has been proved earlier [18]. To measure the transverse conductivity, the films were sandwiched between two contact layers grown by the same method. The thickness of bottom and top layers was close to 30 and $20 \mu \mathrm{m}$, respectively. The top layer of $p^{+}$-PbTe had the area $0.5 \mathrm{~cm}^{2}$. Thereafter, Au metal pads were electrolytically deposited onto the top $p^{+}$-PbTe layer. In the lateral arrangement, the distance between contacts was approximately $2 \mathrm{~mm}$. The photoluminescence measurements were performed at $77 \mathrm{~K}$ under $\mathrm{CW}$ excitation using the $633 \mathrm{~nm}$ line of an He-Ne laser. Two excitation geometries were used in measurements with the laser beam incident onto the free surface and the CdTe/sapphire interface. The laser spot diameter remained unchanged during measurements in order to compare the photoluminescence intensity in samples with different sizes of grains.

\section{Experimental results}

Typical current-voltage characteristics measured for transverse and lateral arrangements of contacts are shown in Fig. 1. As seen, the transverse conductivity is characterized by the linear $I-U$ characteristic that is independent on the polarity of applied voltage. Contrary, the current-voltage characteristic for the lateral conductivity exhibited more complicate behavior, namely: at low bias voltages the linear $I-U$ dependence is observed, followed by the sub-linear one at higher voltages. The non-linear part is fitted by a power-law dependence $I \sim U^{n}$, with the exponent $n \approx 0.5$.

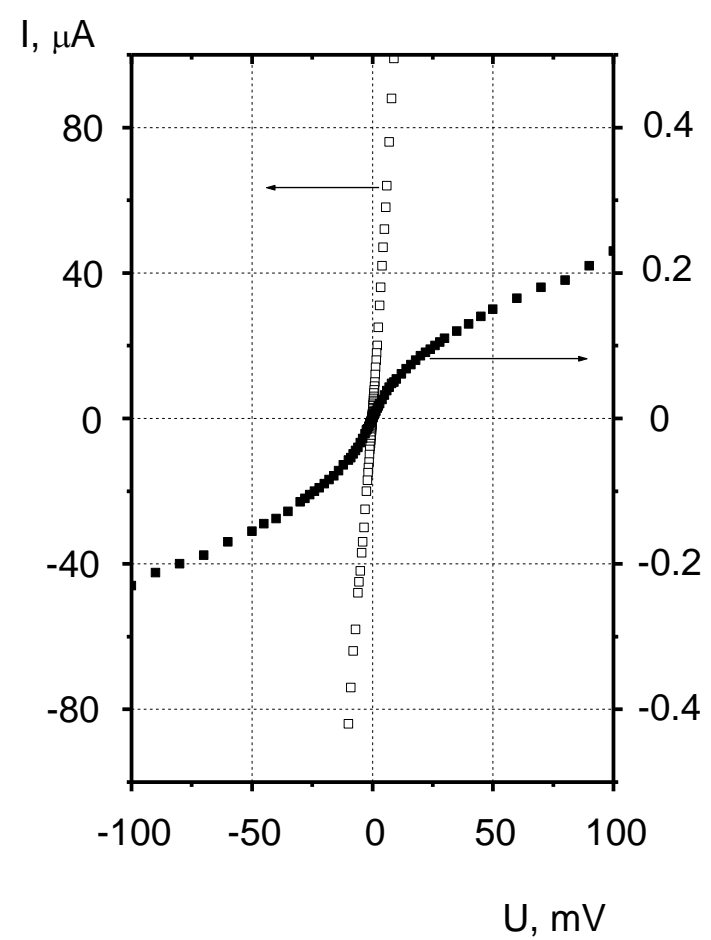

Fig. 1. Current-voltage characteristics for transverse (open dots) and lateral (close dots) arrangement of contacts at room temperature. 
The temperature dependences of the dark current measured at a fixed bias voltage are shown in Fig. 2. For the transverse conductivity, experimental data plotted in co-ordinates $\ln I-1 / T$ are represented by two linear dependences. From their slopes, the activation energies $0.32 \mathrm{eV}$ at $T<220 \mathrm{~K}$ and $0.48 \mathrm{eV}$ at higher temperatures were determined. It is important to note that these energies are independent of the applied voltage. On the contrary, for the lateral conductivity such a dependence is observed, and the activation energy is increased from $0.58 \mathrm{eV}$ at biases $\leq 10 \mathrm{~V}$ to approximately $0.69 \mathrm{eV}$ at the bias voltage $150 \mathrm{~V}$. It is important to note that the activation energy for the lateral conductivity exceeds that for the transverse conductivity.

In principle, in CdTe polycrystalline films two paths for the current flow are possible - across the grains and along the grain boundaries [[19]. In the investigated films, the contribution of grain boundaries to the overall conductivity seems to be negligibly low due to two reasons. First, it is generally believed that grain boundaries are more disordered as compared to those of the intragrain region $[1,2,19]$. This results in a higher resistance of the grain boundary, when comparing to the intragrain regions. Second, because of polycrystalline films investigated in this study are composed of rather large grains, the density of grain boundaries are rather small. The linear current-voltage characteristics observed for the transverse conductivity clearly indicate its ohmic nature due to preferential transport of carriers through the grains. Also, the high-frequency capacitance measured in this structure was found to be determined by the contact geometry and is independent of the polarity of the bias voltage.

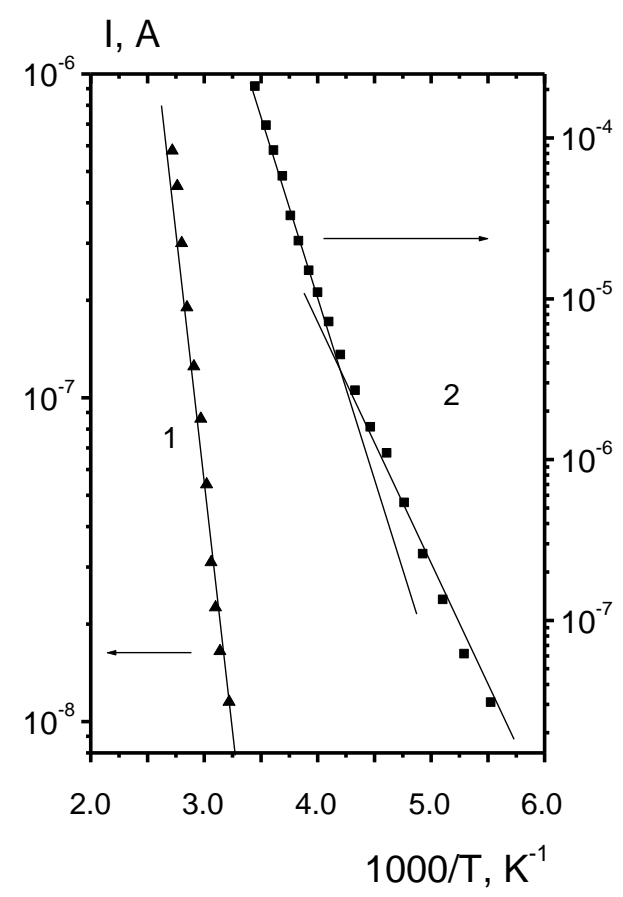

Fig. 2. Temperature dependences of dark current for lateral (1) and transverse (2) arrangement of contacts measured at the bias voltage $10 \mathrm{~V}$.
Two important problems may impede the correct interpretation of transport properties in $\mathrm{CdTe}$ polycrystalline film, namely: uncertainty in the doping level and spatial distribution of charged defects inside the grains. By using the result on the transverse and lateral conductivity measurements, the first problem may be clarified. At room temperature, the transverse conductivity in the investigated films lies within the range $(2 \ldots 6) \cdot 10^{5} \Omega \mathrm{cm}^{2}$. For the carrier mobility $10 \mathrm{~cm}^{2} / \mathrm{V} \cdot \mathrm{cm}$, the hole concentration of the order of $1 \cdot 10^{12} \mathrm{~cm}^{-3}$ was estimated for the intragrain region of a grain. In the calculation, the contact area was assumed to be equal to the physical area of the $p^{+}-\mathrm{PbTe}$ contact pad. This assumption is based on the fact that the lateral conductivity is substantially lower in comparison with the transverse one. For the lateral conductivity, the nonlinear behavior of $I-U$ characteristics indicates existence of potential barriers at the grain boundaries. The increase in the activation energy cannot be explained by the FrenkelPoole emission, since the ionization energy appearing in this case decreases with the applied bias. Such a decrease was observed, for instance, in $\mathrm{ZnO}$ polycrystalline films [20]. To explain this result, a model of the grain boundaries as double Schottky barriers is used. At a low bias voltage, the barrier height for both barriers remains almost unchanged, thus the dark current is determined by the activation energy of defect states in the intragrain region. At higher voltages, the activation energy increases due to enhancement of the reverse biased barrier height.

Since mobile carriers are thermally activated in the intragrain region, the carrier concentration is given by the expression $[2,21]$

$p_{\mathrm{G}} \sim \exp \left(-E_{p} / k T\right)$

where $E_{p}$ is the activation energy of carriers. The conductivity can be written as

$\sigma \sim \exp (-\Delta E / k T)$.

Thus, the relationship between the characteristic energies is

$\Delta E=E_{p}+\varphi_{B}$,

where $\varphi_{B}$ is the barrier height (the band bending) at the grain boundary. Taking into account that $\varphi_{b}=0$ for the transverse conductivity and $E_{p}$ is not changed, the barrier height may be estimated from the difference between the activation energy for the lateral and transverse conductivity. To the authors knowledge, this method for determining the barrier height in CdTe has not been used in application to CdTe polycrystalline films. The potential barrier height within the range $0.2 \ldots 0.3 \mathrm{eV}$ at zero temperature and approximately $0.1 \mathrm{eV}$ at room temperature was obtained. It should be pointed out that the determined values agree well with the data reported earlier [22].

In the Petriz model [2, 3], the barrier height is given by the relation

$q \varphi_{b}=k T \ln \left(p_{G} / p_{G B}\right)$, 
where $p_{G B}$ is the hole concentration in the intergrain (boundary) regions, respectively. For the mean value of $p_{G}=1 \cdot 10^{12} \mathrm{~cm}^{-3}$, the hole concentration trapped at the grain boundary is of the order of $3 \cdot 10^{10} \mathrm{~cm}^{-3}$.

In the above estimation, the spatial distribution of defects is assumed to be uniform. However, it has been previously shown that in the investigated films the grain boundaries are represented by the dislocation network [23]. Taking into account the gettering effect of dislocations in polycrystalline films [24], this result implies inhomogeneous distribution of impurities in the intragrain regions. This conclusion may be referred also to deep defects as well. Their existence has been proved by photoluminescence spectra measurements in the films composed of grains with different sizes, Fig. 3. The observed photoluminescence bands with the peaks at 0.85 and $1.1 \mathrm{eV}$ are attributed to deep defects localized in the intragrain regions. The reason for this assumption is that the intensity of both spectral bands is higher in the films composed of larger grains. In the film with the $10-\mu \mathrm{m}$ grains, the measured signal was too weak to be measured reliably. Also, as seen from Fig. 3, the intensity of the measured spectra depends on the excitation geometry. This result indicates inhomogeneous distribution of deep defects in the intragrain region of grains. Its effect on the barrier height is not clear. However, the pronounced increase in the doping level at the grain boundaries has been found earlier in CdTe polycrystalline films with the barrier height of $0.78 \mathrm{eV}$ [25]. In order to fulfil correct estimations of the barrier height, more sophisticated investigations are needed.

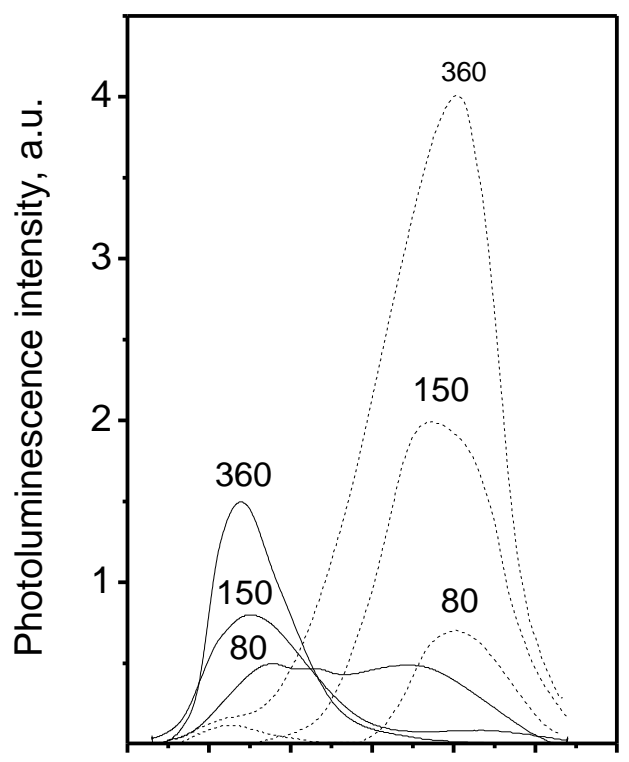

$\begin{array}{lllllll}0.7 & 0.8 & 0.9 & 1.0 & 1.1 & 1.2 & 1.3\end{array}$

\section{Energy, eV}

Fig. 3. Photoluminescence spectra related to deep defects in polycrystalline films with the grain size 80,150 and $360 \mu \mathrm{m}$ measured for the front-side (dashed curves) and back-side (solid curves) excitation.
Deep defect states in undoped CdTe were investigated by many authors, and their energy levels were found in the bandgap of $\mathrm{CdTe}$ by various experimental methods [28-31]. The most important defects are vacancies, interstititals and antisites of both constituents, $\mathrm{Cd}$ and $\mathrm{Te}$, as well as complexes with their participation such as $\mathrm{Te}_{\mathrm{Cd}}-\mathrm{V}_{\mathrm{Cd}}$. However, identification of these levels remains controversial. A most likely that the photoluminescence line at $1.1 \mathrm{eV}$ is caused by recombination of electrons at the acceptor level of doubly ionized cadmium vacancy. Its ionization energy measured from the valence band edge is found to be less than $0.47 \mathrm{eV}[28,29]$. The photoluminescence line at $0.85 \mathrm{eV}$ is related to deeper level at the midgap, and its identification is less clear. Presumably, it may be ascribed to the complex $\mathrm{Te}_{\mathrm{Cd}}-\mathrm{V}_{\mathrm{Cd}}[30]$.

The width of the space charge region related to this barrier is comparable with the grain size or even exceeds it. For the determined value of the barrier height $0.2 \mathrm{eV}$, the space charge region width is ranged from $9 \mu \mathrm{m}$ at zero bias voltage up to $280 \mu \mathrm{m}$ for the bias voltage $100 \mathrm{~V}$. These estimates were obtained for the hole mobility close to $10 \mathrm{~cm}^{2} / \mathrm{V} \cdot \mathrm{s}$ by using the room temperature conductivity values for the transverse arrangement of contacts.

\section{Conclusions}

1. The conductivity is investigated in CdTe polycrystalline films with the columnar structure of grains within the temperature range $218 \ldots 387 \mathrm{~K}$. The transverse (across the film) conductivity is shown to be ohmic in its nature. The concentration of holes in the intragrain and intergrain regions are of the order of $10^{12}$ and $10^{10} \mathrm{~cm}^{-3}$, respectively.

2. The lateral conductivity is dominated by the potential barriers at the grain boundaries. The barrier height is estimated to be of the order of $0.2 \ldots 0.3$ and $0.1 \mathrm{eV}$ at zero and room temperature, respectively.

\section{References}

1. G. Harbeke (Ed.), Polycrystalline Semiconductors. Physical Properties and Applications. Springer, 1985.

2. Lawrence Kazmerski (Ed.), Polycrystalline and Amorphous Thin Films and Devices. Elsevier, 2012.

3. R.L. Petriz, Theory of Photoconductivity in Semiconductor Films // Phys. Rev. 104(6), p. 15081515 (1956).

4. A.Y. Shik, Electronic Properties of Inhomogeneous semiconductors. Gordon and Breach, 1995.

5. A.L. Efros and B.I. Shklovskii, Electronic Properties of Doped Semiconductors. Springer, 1992.

6. A.V. Sukach, V.V. Tetyorkin and N.M. Krolevec, Mechanisms of carrier transport in CdTe polycrystalline films // Semiconductor Physics, Quantum Electronics and Opoelectronics, 13(3), p. 254-260 (2010). 
7. T.M. Razykov, C.S. Ferekides, D. Morel, E. Stefanakos, H.S. Ullal, H.M. Upadhyaya. Solar photovoltaic electricity: Current status and future prospects // Solar Energy, 85(8), p. 1580-1608 (2011).

8. A. De Vos, J.E. Parrott, P. Baruch, P.T. Landsberg, 12th European Photovoltaic Solar Energy Conf., Amsterdam, The Netherlands, p. 1315, 1994.

9. T. Aramoto, S. Kumazawa, H. Higuchi et al., $16.0 \%$ efficient thin-film CdS/CdTe solar cells // Jpn. J. Appl. Phys. 36(10), p. 6304-6305 (1997). $\mathrm{X}$. Wu, High-efficiency polycrystalline CdTe thinfilm solar cells // Solar Energy, 77, p. 803-814 (2004).

10. M. Hage-Ali and P. Siffert, Semiconductors for room temperature nuclear detector applications in: Semiconductor and Semimetals, Vol. 43, Eds. T.E. Schlesinger, and R.B. James. Academic Press, San Diego, 1995.

11. Kevin D. Dobson, Iris Visoly-Fisher, Gary Hodes and David Cahen, Stability of CdTe/CdS thin-film solar cells // Solar Energy Materials and Solar Cells, 62(3), p. 295-325 (2000).

12. T.L. Chu, S.S. Chu, Thin film II-VI photovoltaics // Sol.-State Electron. 38(3), p. 533-549 (1995).

13. T.A. Gessert, S. Asher, S. Johnston, M. Young, P. Dippo, C. Corwine, Analysis of $\mathrm{CdS} / \mathrm{CdTe}$ devices incorporating a $\mathrm{ZnTe}: \mathrm{Cu} / \mathrm{Ti}$ contact // Thin Solid Films, 515(15), p. 6103-6106 (2007).

14. B. Ghosh, S. Purakayastha, P.K. Datta, R.W. Miles, M.J. Carter, R. Hill, Formation of a stable ohmic contact to CdTe thin films through the diffusion of P from Ni-P // Semicond. Sci. Technol. 10(1), p. 7176 (1995).

15. N. Romeo, A. Bosio, R. Tedeschi, A. Romeo, V. Canevari, A highly efficient and stable $\mathrm{CdTe} / \mathrm{CdS}$ thin film solar cell // Solar Energy Materials \& Solar Cells, 58, p. 209-218 (1999).

16. D.L. Bätzner, A. Romeo, M. Terheggen, M. DÖbeli, H. Zogg, A.N. Tiwari, Stability aspects in CdTe/CdS solar cells // Thin Solid Films, 451452, p. 536-543 (2004).

17. H. Abrams, Grain size measurement by the intercept method // Metallography, 4(1), p. 59-78 (1971).

18. A.V. Sukach, V.V. Tetyorkin, A.I. Tkachuk, Heterostructure ohmic contacts to p-CdTe polycrystalline films // Semiconductor Physics, Quantum Electronics and Opoelectronics, 17(3), p. 268-271 (2014).

19. R. Labusch and J. Hess, Conductivity of grain boundaries and dislocations in semiconductors, in:
Point and Extended Defects in Semiconductors (G. Benedekt, Ed.) // NATO ASI Series, 202, p. 1537 (1989).

20. Mohammad A. Alim, Shengtao Li, Fuyi Liu, and Pengfei Cheng, Electrical barriers in the $\mathrm{ZnO}$ varistor grain boundaries // phys. status solidi $(a)$, 203(2), p. 410-427 (2006).

21. R.K. Mankarious, Hall mobility measurements in CdS films // Solid-State Electron. 7(9), p. 702-704 (1964).

22. S.A. Kolosov, Y.V. Klevkov, A.F. Plotnikov, Transport phenomena in coarse-grained polycrystals of CdTe // Semiconductors, 38(3), p. 293-297 (2004).

23. V.V. Tetyorkin, A.V. Sukach, S.V. Stariy and V.A. Boiko, Photoluminescence studies of CdTe polycrystalline films // Semiconductor Physics, Quantum Electronics and Opoelectronics, 15(4), p. 340-344 (2012).

24. D.B. Holt and B.G. Yacobi, Extended Defects in Semiconductors. Electronic Properties, Device Effects and Structures. Cambridge University Press, 2007.

25. A.S. Gilmore, V. Kaydanov, T.R. Ohno, D. Grecu, and D. Rose, Impedance spectroscopy and Hall measurements on CdTe thin polycrystalline films, in: II-VI Compound Semiconductor Photovoltaic Materials, MRS Proc., 668 (2001).

26. P. Capper (Ed.), Properties of Narrow Gap Cadmium-based Compounds. Institution of Electrical Engineering, London, 1994.

27. R. Triboulet, P. Siffert, CdTe and related compounds: physics, defects, hetero- and nanostructures, crystal growth, surface and applications - Part I: Physics, CdTe-based Nanostructures, Semimagnetic Semiconductors, Defects. Elsevier, 2010.

28. B.K. Meyer, P. Omling, E. Weigel, and G. Mullervogt, F-center in CdTe // Phys. Rev. B, 46(23), p. 15135-15138 (1992).

29. P. Emanuelsson, P. Omling, B.K. Meyer, M. Wienecke, and M. Schenk, Identification of the cadmium vacancy in CdTe by electronparamagnetic resonance // Phys. Rev. B, 47(23), p. 15578-15580 (1993).

30. R. Soundararajan, K.G. Lynn, S. Awadallah, C. Szeles, and S.H. Wei, Study of defect levels in CdTe using thermoelectric effect spectroscopy // J. Electron. Mat. 35(6), p. 1333-1340 (2006).

31. A. Castaldini, A. Cavallini, B. Fraboni, P. Fernandez, and J. Piqueras, Deep energy levels in CdTe and CdZnTe // J. Appl. Phys. 83(4), p. 2121-2126 (1998). 